ISSN: 2602-8506

Vol. 3, N³., p. 100-116, julio - septiembre, 2019

Recibido: 03-05-2019/Aceptado: 03-06-2019/ Publicado: 03-07-2019

\title{
Modelo de gobierno electrónico peruano: servicios para el ciudadano en los portales de los gobiernos regionales
}

\author{
Evaluation of services for the citizen in the portals of the regional \\ governments, according to the model of peruvian electronic government \\ Pablo Felipe Alvarado Aguirre. ${ }^{1}$, Paolo Israel Cevallos Vásquez. ${ }^{2}$, Edwin Daniel Moran \\ Villamarin. ${ }^{3} \&$ Lenin Feliz Preciado Ortiz. ${ }^{4}$
}

\section{DOI: https://doi.org/10.33262/visionariodigital.v3i3.656}

\begin{abstract}
.
This research starts with a minimal introduction that we want to achieve in this work. In the second chapter, we incorporate the main laws related to the citizen's services in The Public Administration, those laws will allow us to understand the basic legal of State Modernization's Process, E-Government, Institutional Web Sites and The Government Regionals of Perú. We briefly describe about the antecedents, the definitions, and the progress of government in Perú and The World. The fourth chapter is divided into two parts, the first part describes about the special measures denominated «criterion », we also described and selected the Methodology, these elements will allow The Global Evaluation of Web Sites of (25) Government
\end{abstract}

\footnotetext{
${ }^{1}$ Universidad Técnica Luis Vargas Torres, Sede Santo Domingo, La Concordia, Ecuador. Facultad de Ciencias Administrativas. Universidad de las Fuerzas Armadas - ESPE. pfalvarado@ hotmail.com

${ }^{2}$ Universidad Técnica Luis Vargas Torres, Sede Santo Domingo, La Concordia, Ecuador. Facultad de Ciencias Administrativas. cevallos_paolo@yahoo.es

3 Universidad Técnica Luis Vargas Torres, Sede Santo Domingo, Santo Domingo - Ecuador. Facultad de Ciencias Administrativas. dani19812009@live.com

${ }^{4}$ Universidad Técnica Luis Vargas Torres, Sede Santo Domingo, Santo Domingo - Ecuador. Facultad de Ciencias Administrativas. preciadolenin@gmail.com
} 
ISSN: 2602-8506

Vol. 3, N³., p. 100-116, julio - septiembre, 2019

Regionals, for this evaluation we don't considerate the back office; the next part shows the final results that are subdivided into three parts: 1) description of egovernment development's actual state in the 25 Government Regionals, 2) detail the features related to interaction, information and digital services and 3) description of the current states of some services for the citizens, such as online access information, online procedures and online participation.

Keywords: E-Government, Citizen's Services, Web Sites.

\section{Resumen.}

La investigación inicia con una parte introductoria de lo que se pretende realizar en el presente trabajo. En el segundo capítulo se incorpora la normativa relativa al servicio del ciudadano en la Administración Pública, los cuales permiten entender la legislación básica del proceso de Modernización de la Gestión del Estado, el Gobierno Electrónico, los Portales Web y los Gobiernos Regionales en el Perú. Se hace una breve contextualización de los antecedentes y avances de Gobierno Electrónico en el mundo y en el Perú. El cuarto capítulo se divide en dos partes, en el primero se realiza la selección de criterios y la metodología que permitirán evaluar de manera global el front office de los portales web de cada Gobierno Regional y en la segunda parte se exponen los resultados obtenidos, que a su vez: 1) muestra el estado actual de desarrollo de Gobierno Electrónico en los 25 gobiernos regionales del Perú, 2) detalla las características relacionadas a la interacción, información y medio digital y 3) describe el estado actual de algunos servicios para el ciudadano como el acceso a la información, los trámites en línea y la participación.

Palabras claves: Gobierno Electrónico, Servicios al Ciudadano, Portal Web, Gobiernos Regionales del Perú y Administración Pública 


\section{Introducción}

El Gobierno Electrónico es un concepto ligado al uso de las tecnologías de información y comunicaciones para mejorar las actividades y la calidad del servicio al ciudadano en las administraciones públicas. Uno de los retos que asume la mayoría de las administraciones públicas en el mundo es proporcionar un mayor dinamismo entre el Estado y los ciudadanos, con el fin de lograr el buen Gobierno, una forma de gobierno que se busca desde la antigua Grecia.

El Gobierno Electrónico es un aspecto clave en el proceso de modernización del Estado peruano, promueve el acercamiento de la administración pública a la población a través del uso de las tecnologías de información y comunicaciones para asegurar: el acceso a la información, los trámites por medios electrónicos, la transparencia de los asuntos públicos y la participación dinámica de la ciudadanía. El Gobierno Electrónico alcanza a todas las entidades públicas que conforman el Estado peruano, el cual es impulsado e implementado bajo la supervisión de la Presidencia de Consejo de Ministros del Perú desde el año 2003. De acuerdo con los datos que brinda las Naciones Unidas en su sitio web, en el 2016, el Reino Unido de Gran Bretaña e Irlanda del Norte ocupa el primer lugar en el índice de desarrollo de e-Government, mientras que Perú ocupa el puesto 81 aunque en el 2014 se ubicaba en el puesto 72, datos que hacen suponer que el Gobierno Electrónico en el Perú no es efectivo en su desarrollo. La eficiencia en las administraciones públicas modernas automáticamente genera ideas relacionadas con la calidad de servicio que recibe un ciudadano en un medio electrónico, a raíz de ello, en el plano nacional e internacional hay estudios que se enfocan en la evaluación de portales web de administraciones, municipios o universidades en base a su marco de Gobierno Electrónico, de tal modo que llegan a responder preguntas como: ¿qué servicios online se ofrece al ciudadano en los portales web?, ¿cuáles son las características principales de los servicios en los portales web? o ¿cuál es el estado actual de desarrollo de Gobierno Electrónico? lamentablemente en el Perú no hay estudios de investigación visibles o informes globales que tome en consideración a los Gobiernos Regionales y que además respondan a dichas cuestiones. 
En los portales web institucionales del Registro Nacional de Identificación y Estado Civil, la Superintendencia Nacional de Aduanas y de Administración Tributaria y el Organismo Supervisor de Contrataciones del Estado se observa que se han habilitado diferentes medios de interacción para el ciudadano e intentan cumplir con los lineamientos propuestos por el Gobierno Electrónico peruano; en cambio, es difícil afirmar esta posición cuando se trata de un Gobierno Regional, pese a que este nivel de Gobierno tiene la potestad de elaborar y ejecutar programas de desarrollo integral. En el Perú, algunos estudios demuestran que se han realizado evaluaciones de Gobierno Electrónico de manera local e institucional, un ejemplo de ello, es el caso de la Autoridad Nacional del Servicio Civil, que en el año 2016 elaboró su diagnóstico de Gobierno Electrónico tomando en cuenta el modelo de nivel de madurez de desarrollo de Gobierno Electrónico propuesto por las Naciones Unidas, para ello generó sus propios criterios de evaluación y concluyó de que se encontraba entre la etapa de "ampliada" e "interactiva". A enero del 2018, fecha de inicio del presente trabajo, no se encontró investigaciones referentes a evaluaciones que muestren de manera global o conjunta el desarrollo de gobierno electrónico en los gobiernos regionales, o estudios que describan las características y/o servicios que se ofrecen en el portal web de cada Gobierno Regional, servicios que en teoría cumplen las necesidades del ciudadano y están alineados a la Política de Gobierno Electrónico Peruano del 2013-2017 ya que en el 2017el Gobierno Central declaró que era el "Año del Buen Servicio al Ciudadano" (Presidencia de Consejo de Ministros, 2017, p. 1). De ahí que, tomando en cuenta el front office de los portales web institucionales y desde la percepción de una ciudadana, el presente trabajo pretende solventar la falta de información y dar a conocer primero el estado actual de desarrollo de Gobierno Electrónico en los gobiernos regionales, en segundo lugar evidenciarlas características principales de los servicios en los portales web de los gobiernos regionales y por último describir algunos aspectos relativos a los servicios que se ofrecen al ciudadano en dichos portales de los gobiernos regionales, servicios que están orientados a promover la transparencia, la participación y la agilización de trámites administrativos los que a su vez están alineados a la Política Nacional de Modernización de la Gestión Pública en el Perú. 
ISSN: 2602-8506

Vol. 3, N³., p. 100-116, julio - septiembre, 2019

Por lo tanto, El objetivo general de esta investigación es evaluar los servicios para el ciudadano en los portales de los veinticinco (25) gobiernos regionales, según el modelo de Gobierno Electrónico peruano.

\section{Marco Teórico}

El internet nace en los 60 en Estados Unidos con el Advanced Research Project Agency Network como parte de la respuesta gubernamental a Sputnik de la Unión Soviética durante la guerra fría, "cuya finalidad era, no depender de un ordenador central" (Álvarez Barrios, 2012, p. 19), en los 90 aparece el primer navegador que solía ser utilizado por pequeñas elites conocido por algunos como "digeratis" (Castell, n.d.), término que se utiliza de manera humorística al referirse a las personas que conocen y utilizan bastante las computadoras e internet.

En 1993, el presidente Clinton y el vicepresidente Gore de los Estados Unidos, proponen reinventar el Gobierno Federal bajo la misión de crear un Gobierno que trabaje mejor, a un coste menor y con resultados favorables para los estadounidenses, el reinvento duro casi una década, la visión era cambiar la experiencia del Ciudadano-Gobierno a través de las Tecnologías de Información y Comunicaciones en adelante Tics, cuya visión además se orientaba a que, tanto la información personal como los servicios sean controladas según las necesidades del ciudadano y no por los gobiernos (Kamensky, 1999), todas estas iniciativas trajeron el concepto de gobierno electrónico en el mundo.

El modelo tradicional de la administración pública se ha caracterizado por ser estática, por estar orientada a sí misma, de comunicación jerárquica y regulada por normas rígidas; sin embargo, hace más de dos décadas los gobiernos vienen promoviendo una administración mucho más orientada al ciudadano, una que sea inversamente proporcional al modelo clásico de Max Weber, estanueva orientación busca brindar mayor interactividad y disposición de parte de las administraciones hacia los ciudadanos con el uso de las tecnologías, donde no solo ciudadanos sino los gobiernos se beneficien con las ventajas que esta trae consigo. Estados Unidos, China, Japón, Alemania y Reino Unido 
ISSN: 2602-8506

Vol. 3, N³., p. 100-116, julio - septiembre, 2019

(UK) son países económicamente desarrollados en el mundo, sin embargo, no todos ellos ocupan un buen lugar en el ranking de desarrollo de Gobierno Electrónico, en la Figura 1 podemos observar a los 10 países que lideran en el desarrollo de e-government, los cuales se enfocan en reforzar la participación ciudadana, crear nuevos servicios online, capital humano e infraestructura de telecomunicaciones para interactuar mejor con el ciudadano.

Figura 1. Índice de desarrollo de Gobierno Electrónico. Fuentes: (Naciones Unidas, 2016).

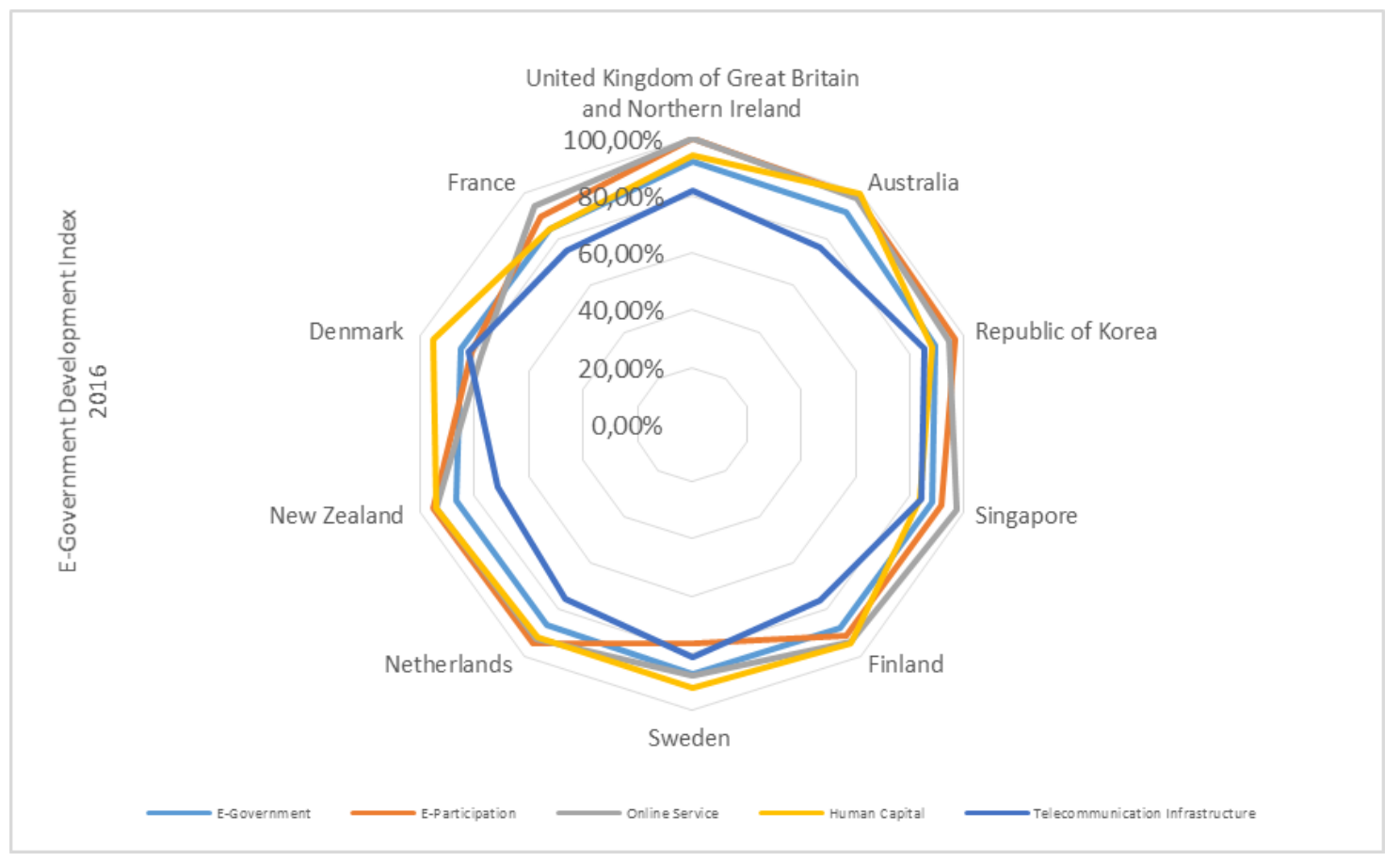

Elaborado por: Los autores

\section{Definición, categoría y focalización de Gobierno Electrónico}

Mesa Torre, autor de la tesis "Interoperabilidad como parte del Desarrollo del Gobierno Electrónico en el Perú" menciona que para: la Organización de las Naciones Unidas (ONU) el Gobierno Electrónico es la utilización de Internet y el World Wide Web para entregar información y servicios del Gobierno a los ciudadanos; la Unión Internacional de Telecomunicaciones (UIT) el Gobierno Electrónico es el uso de las tecnologías de la información y comunicación para prestar servicios públicos, mejorar la eficacia gerencial y promover valores democráticos, así como un marco regulatorio que facilite información 
para iniciativas intensivas y promueva la Sociedad de la Información; y la Organización para la Cooperación y el Desarrollo Económico en adelante OCDE, el Gobierno Electrónico es la oportunidad de desarrollar una nueva relación entre el Gobierno, ciudadanos, usuarios de servicios y empresas, a través de las TIC, permitiendo la difusión y recopilación de información y servicios tanto dentro como fuera del Gobierno para la prestación de servicios, la toma de decisiones y la rendición de cuentas (2014).

\section{De Gobierno Electrónico a Gobierno Abierto}

Desde el 2005 los países han incorporado en sus estrategias de Gobierno Electrónico la información en línea; la georreferenciación; la simplificación administrativa; la desmaterialización de procedimientos; la firma electrónica y digital; los registros, documentos y archivos electrónicos; la preservación de la información; la interoperabilidad; la protección de datos; los medios de pago electrónico; la seguridad, usabilidad, accesibilidad; la contratación electrónica; las ventanillas únicas entre otras, y en la última década algunos países muestran mayor disposición de entregar información y datos de interés público a la sociedad (Naser \& Hofmann, 2016, p. 23).

\section{El Gobierno Electrónico Peruano}

El Gobierno Electrónico Peruano busca: mejorar la gobernabilidad a través de la generación de valor público para los ciudadanos, apoyar a las instituciones del ámbito local, regional o nacional en el cumplimiento de sus metas institucionales, sean estas intrainstitucionales o interinstitucionales y se encuentra alineado a las tendencias mundiales. El modelo de nivel de madurez por etapas de desarrollo en materia de Gobierno Electrónico, el cual nos permitirá realizar una aproximación global del nivel de government-to-citizen desarrollado en las administraciones de los Gobiernos Regionales, se debe remarcar para la selección de los criterios de evaluación, se toma en cuenta el front office de los portales web y los tres lineamientos del modelo de Gobierno Electrónico peruano.

\section{Indicadores para evaluar el grado de desarrollo de Gobierno Electrónico.}

Las etapas de desarrollo del modelo propuesto por la Organización de las Naciones Unidas del Instituto Latinoamericano del Planificación, Económica y Social - Comisión 
ISSN: 2602-8506

Vol. 3, N³., p. 100-116, julio - septiembre, 2019

Económica para América Latica y el Caribe que se muestra en el estudio de Gobierno Electrónico en la Gestión Pública realizada por (Naser \& Concha, 2011) es la que mejor se adapta al presente trabajo; también es necesario mencionar que este modelo se utilizó en el diagnóstico realizado en el 2016 por la Autoridad Nacional del Servicio Civil, en adelante SERVIR, con el que se evaluaron aspectos que recaen sobre gobierno electrónico; para dicha evaluación, SERVIR generó sus propios atributos que servirán de referencia en la elaboración de los indicadores que corresponden al presente trabajo.

Tabla 1. Descripción y atributos de nivel de madurez de iniciativas de Gobierno Electrónico

\begin{tabular}{|c|c|c|c|}
\hline $\begin{array}{l}\text { Nivel de } \\
\text { madurez }\end{array}$ & Descripción & Atributos & $\%$ \\
\hline Emergente & $\begin{array}{l}\text { El país o la entidad asume el compromiso de } \\
\text { desarrollar Gobierno Electrónico, pero sólo } \\
\text { información básica se brinda a través de } \\
\text { Internet. }\end{array}$ & $\begin{array}{l}\text { Logo, mapa } \\
\text { sitio, contactos }\end{array}$ & $0-20$ \\
\hline Ampliada & $\begin{array}{l}\text { La presencia en línea del país se expande. Crece la } \\
\text { cantidad de sitios Web, y se provee interacción a través } \\
\text { de medios más sofisticados (búsqueda en sitios Web e } \\
\text { interacción a través de } \\
\text { correo electrónico }\end{array}$ & Mapas, buscador & $21-40$ \\
\hline Interactiva & $\begin{array}{l}\text { Existe una presencia masiva de servicios con } \\
\text { interacción más sofisticada, por ejemplo, } \\
\text { llenado y envío de formularios electrónicos }\end{array}$ & $\begin{array}{l}\text { Formularios } \\
\text { electrónicos }\end{array}$ & $41-60$ \\
\hline Transaccional & $\begin{array}{l}\text { El Estado o la entidad ofrece transacciones completas } \\
\text { y seguras tales como: obtención de } \\
\text { visas y pasaportes, certificados de nacimiento y } \\
\text { defunción, pago de multas e impuestos, etc. }\end{array}$ & $\begin{array}{l}\text { Trámites en línea, } \\
\text { certificado digital, pagos } \\
\text { en línea. }\end{array}$ & $60-80$ \\
\hline Integración & $\begin{array}{l}\text { Acceso instantáneo a servicios de manera integrada. } \\
\text { El usuario no percibe las fronteras entre los distintos } \\
\text { servicios }\end{array}$ & $\begin{array}{l}\text { Participación } \\
\text { ciudadana, ventanilla } \\
\text { única, } \\
\text { gobierno abierto }\end{array}$ & $81-100$ \\
\hline
\end{tabular}

Fuente: Elaboración propia en base al modelo de las Naciones Unidas y Servir 
ISSN: 2602-8506

Vol. 3, N³., p. 100-116, julio - septiembre, 2019

\section{Metodología.}

La metodología del INCAE clasifica a tres las categorías de calidad, las cuales se subdividen en 6 niveles y estas en 24 criterios generales y a cada uno de estos criterios se le asigna pesos, los cuales detallaremos en las siguientes tablas.

El significado de los pesos según Robayo Valencia (2017, p. 36) se detalla de la siguiente manera:

Peso 1. Acciones básicas sin necesidad de interacción.

Peso 2. Requiere de interacción lo que puede involucrar cambios en los procesos de la institución.

Peso 3. Implica el desarrollo de una infraestructura compleja, que permita la interoperabilidad entre instituciones.

Para la calificación de cada uno de los indicadores, los cuales hacen un total de 52, también se le asigna un valor categórico a cada indicador, es decir, si cumple con el indicador se le asigna un " 1 " y si no cumple con el indicador se le asigna un " 0 ".

El método matemático por el que se obtiene la nota final de la evaluación es mediante la siguiente formula:

$$
\text { Nota Fual }=\sum_{i=1}^{24} w_{i} \frac{x_{i}}{n_{i}}
$$

Donde i es la cantidad de criterios presentes en la metodología, $x_{i}$ es la calificación obtenida en un criterio determinado, $n_{i}$ el peso asignado a un criterio determinado y $W_{i}$ es el valor ponderado de los 24 criterios, tomando, además, en consideración los pesos propuestos en la metodología del INCAE, los que a su vez detallamos a continuación en las tablas 5, 6 y 7. 
ISSN: 2602-8506

Tabla 2. Criterios para evaluar la calidad de la información

\begin{tabular}{ll}
\hline Criterio & Peso \\
\hline Presentación & 2 \\
Transacción simple & 3 \\
Transacción compleja & 3 \\
Integración & 3 \\
Organización - perfil & 2 \\
Integración - perfil & 2 \\
Personalización & 2 \\
Personalización inteligente & 2 \\
Total, Pesos & 19 \\
\hline
\end{tabular}

Fuente: Elaboración propia

\section{Resultados.}

\section{De la calidad de sus principales características}

Los resultados obtenidos respecto a las tres (3) categorías se muestran en la figura siguiente.

Figura 2. Nota final de las tres categorías de calidad

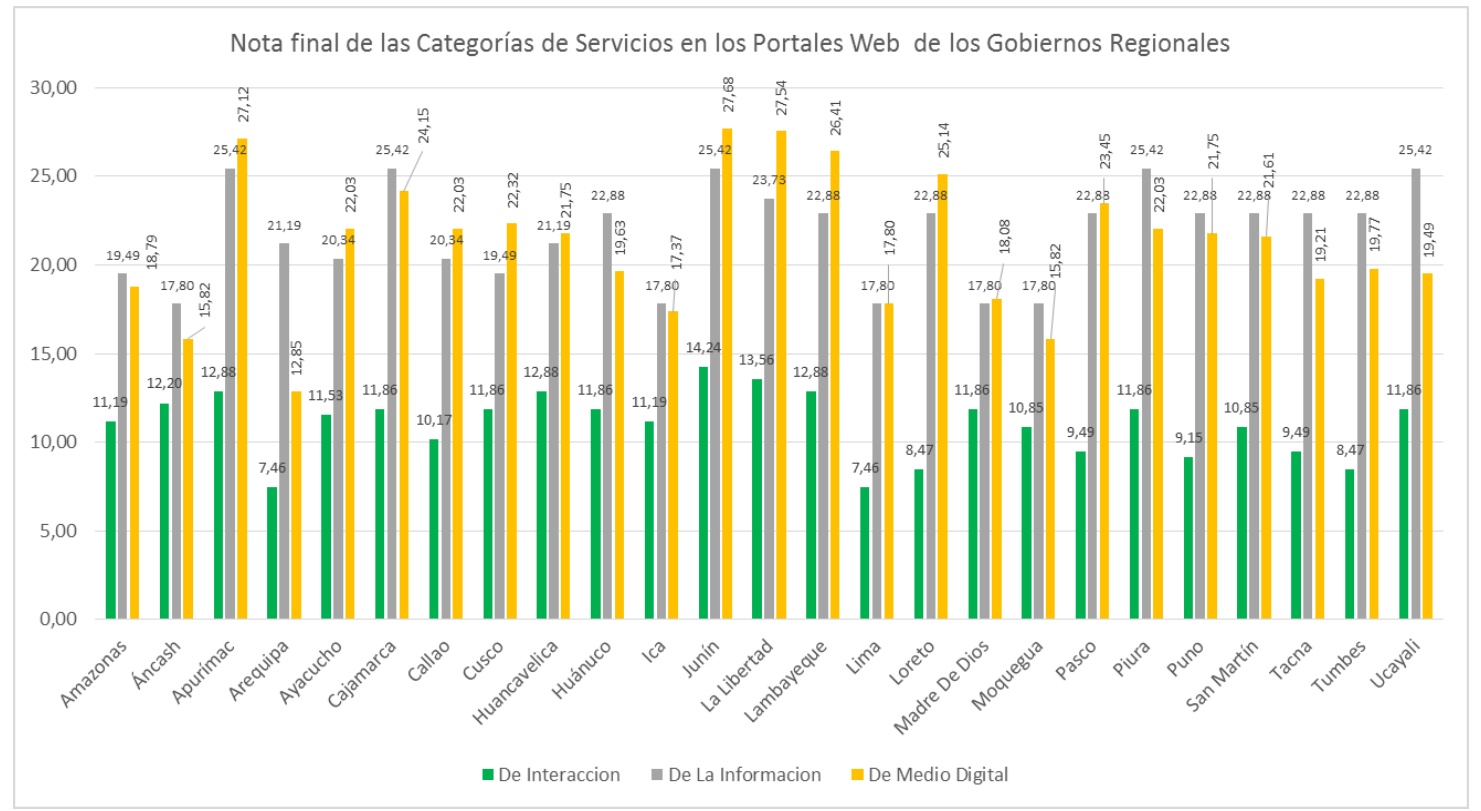

Fuente: Elaboración propia. 
ISSN: 2602-8506

Vol. 3, N³., p. 100-116, julio - septiembre, 2019

Categoría de calidad de interacción: bajo esta categoría los gobiernos regionales de Ancash, Apurímac, Huancavelica, Lambayeque, La libertad y Junín han obtenido las notas más altas que oscilan entre 12,20 a 14,24 de los cuales el primer lugar ocupa el portal web del Gobierno Regional Junín, en tanto hay otras regiones que han obtenido 7,46 ; al margen del puntaje obtenido por cada uno de los 25 gobiernos regionales, es importante resaltar las características encontradas en cada uno de los criterios que pertenecen a esta categoría: Presentación, Transacción simple, Transacción compleja, Organización.

Categoría de calidad de la información: Los gobiernos regionales de La Libertad, Apurímac, Cajamarca, Junín, Piura y Ucayali han obtenido las notas más altas que oscilan entre 23,73 a 25,42 mientras que la nota más baja está sobre los 17,80; algunas características encontradas en cada uno de los criterios que pertenecen a esta categoría son: Comprensividad y Exactitud, Claridad, Correcto y Conciso, Aplicabilidad, Consistente, Actual.

Categoría de medio digital: bajo esta categoría los gobiernos regionales de Cajamarca, Loreto, Lambayeque, Apurímac, La Libertad, Junín han obtenido las notas más altas que oscilan entre 24,15 a 27,68. Algunas de las características encontradas en esta categoría son: Navegabilidad, Flexibilidad, Verificabilidad, Usabilidad, Accesibilidad, Seguridad y Visibilidad para motores de búsqueda, Velocidad.

De todo lo descrito podemos inferir, de que las principales características de los portales web por medio del cual se ofrecen servicios al ciudadano en los gobiernos regionales tiene falencias notorias en la categoría de la calidad de interacción, esta categoría puede hacer que la experiencia del ciudadano resulte poco agradable cuando intenta interactuar con los portales web de los gobiernos regionales, mientras que debemos rescatar que las otras dos categorías de calidad es decir, la de información y de medio digital están a un nivel promedio de aceptación, sin embargo las mejoras deben ser continuas. 


\section{Conclusiones}

Los servicios habilitados para el ciudadano, observados desde el front office aún son incipientes en la gran mayoría de los gobiernos regionales con algunas pocas excepciones, ya que no se promueve de manera íntegra la simplificación de los procedimientos administrativos $\mathrm{u}$ otros servicios digitales que puedan cubrir las necesidades de los ciudadanos, pese a que tanto la política de Gobierno Electrónico y la Política de Modernización del Estado han planteado como uno de sus objetivos pilares el servicio al ciudadano.

La evaluación de los portales web institucionales, permitió describir algunas características de la calidad de interacción, calidad de información y calidad de medio digital, que deben ser mejoradas por la gran mayoría de los gobiernos regionales, ya que se ha evidenciado que en la actualidad no se entregan servicios de calidad al ciudadano. De lo resultados se resalta que el portal de transparencia suma puntos a la categoría de calidad de información, sin embargo, no todos los gobiernos regionales cumplen con publicar y actualizar la información que se produce en cada administración, ya que, si se cumpliese con ello, esto permitiría que los gobiernos regionales fortalezcan su legitimidad, transparencia, participación ciudadana y estarían a un paso de convertirse en gobiernos abiertos.

No debemos olvidar que el Gobierno Electrónico es el pilar del proceso de Modernización del Estado, y este proceso está ligado con la nueva forma de gestionar el Estado (buen gobierno) a través de las tecnologías de información y comunicaciones para que la relación de ciudadano y gobierno sea satisfactoria, se ha visto que muchos gobiernos han adoptado diferentes mecanismos de mejora, pero, la diferencia principal y real entre el Perú y otros países líderes en Gobierno Electrónico radica en que todos ellos se enfocan en mejorar continuamente la funcionalidad, el contenido y la estructura de sus portales web de acuerdo a las necesidades de sus ciudadanos y consecuentemente han obtenido resultados positivos en materia de Gobierno Electrónico. 
ISSN: 2602-8506

Vol. 3, N³., p. 100-116, julio - septiembre, 2019

Sabiendo que la estructura, el contenido y la funcionalidad de los portales web en los gobiernos regionales es un factor condicionante en el largo camino del desarrollo del Gobierno Electrónico, además de ser un medio digital importante en el que: por un lado se ofrece servicios, trámites información y por otro lado se promueve la transparencia y la participación a las distintas generaciones de "baby boomers", "x" y " millenians" de nuestro querido Perú, es importante que los Gobiernos Regionales repliquen algunas de las iniciativas propuestas en el presente trabajo ya que con ello se podría generar economías de escala, educar a los ciudadanos y consiguientemente se podría reducir la brecha geográfica y digital que en la actualidad existe

\section{Referencias bibliográficas.}

Álvarez Barrios, J. E. (2012). Gobierno electrónico: ¿mito o realidad? análisis, descripción y evaluación de los Sitios Web Gubernamentales de las 32 Entidades Federativas. México: UNAM. Recuperado de https://bit.ly/2tuyKOK

Barahona, J. C., \& Elizondo, A. M. (2015). Evaluación de la calidad de la prestación de servicios públicos por medios digitales en Costa Rica. Costa Rica: INCAE Business School. Recuperado de https://bit.ly/2Mevi2G

Bose, R. (2004). Information Technologies for Education \& Training in E-Government.

Alburqueque: IIEE Computer Society. Recuperado de https://bit.ly/2MOgglx Castells, M. (n.d.). Internet y la Sociedad Red. Recuperado de https://bit.ly/2I2Qdo2

Congreso de la República. (2002). Ley Marco de Modernización de la Gestión del Estado. Lima, Perú: Diario Oficial El Peruano. Recuperado de https://bit.ly/2BmCdBg

Congreso de la República. (2007). Ley Orgánica del Poder Ejecutivo. Lima, Perú: Diario Oficial El Peruano. Recuperado de https://bit.ly/2MLScj5

Congreso de la República. (2015). Ley de Promoción de la Banda Ancha y Construcción de la Red Dorsal Nacional de Fibra Óptica. Lima, Perú: Ministerio de Justicia. Recuperado de https://bit.ly/2Kc70cd

Contraloría General de la República. (2014). Estudio del proceso de descentralización en el Perú. Lima: Contraloría General de la República. Recuperado de https://bit.ly/1IEVUBA 
Galindo, F., \& Mezzaroba, O. (2010). Democracia electrónica. Zaragoza: Prensas Universitarias de Zaragoza. Recuperado de https://bit.ly/2MPmql0

Huawei. (2017). Global Connectivity Index. Recuperado Abril 20, 2018, de https://bit.ly/2qXVI6z

Kamensky, J. (1999). A Brief History. Recuperado April 12, 2018, de https://bit.ly/2losR20

Kim, B. J., Oh, K.-T., Han, B.-H., Park, S.-J., \& Lyu, H.-S. (2016). ¿Why Digital Government Not e-Government?:The Paradigm Shift of D.gov in Korea (pp. 530-531). Shanghai, China: ACM Digital Library. https://doi.org/10.1145/2912160.2912233

Kumar, A., \& Joshi, A. (2017). Ontology Driven Sentiment Analysis on Social Web for Government Intelligence (pp. 134-139). New Dheli AA, India: ACM Digital Library. https://doi.org/10.1145/3055219.3055229

Mesa Torre, C. (2014). La interoperabilidad como parte del desarrollo del Gobierno Electrónico en el Perú. Lima: Pontificia Universidad Católica del Perú. Recuperado de https://bit.ly/2yy9zAF

Mesia Rios, J. (n.d.). Proyectos Regionales, hacia una sociedad de la información y el conocimiento. FITEL. Recuperado de https://bit.ly/2IeipmI

Ministerio de Justicia y Derechos Humanos. (2017). Decreto Supremo que aprueba el Texto Único Ordenado de la Ley $\mathrm{N}^{\circ} 27444$ - Ley del Procedimiento Administrativo General. Lima, Perú: Diario Oficial El Peruano.

Naciones Unidas. (2016). E-Government Development Index. Recuperado febrero 23, 2018, de https://bit.ly/2bxIEpE

Naser, A., \& Concha, G. (2011). El gobierno electrónico en la gestión pública. Santiago de Chile: CEPAL-Naciones Unidas. Recuperado de https://bit.ly/2MJvEPM

Naser, A., \& Hofmann, A. (2016). La contribución del gobierno electrónico y los datos abiertos en la integración regional. Santiago: CEPAL-Naciones Unidas. Recuperado de https://bit.ly/2Mfg0e9

OCDE. (2001). Understandin The Digital Divide. Paris: Organización de Cooperación y Desarrollo Económico. Recuperado de https://bit.ly/2qGJAT9

ONGEI. (2012a). Estrategia Nacional de Gobierno Electrónico 2013-2017. Presidencia de Consejo de ministros. Recuperado de https://bit.ly/2K537Tb 
ONGEI. (2012b). Plan Nacional de Gobierno Electrónico 2013 - 2017. Lima: Presidencia de Consejo de Ministros. Recuperado de https://bit.ly/2K537Tb

Ortega Santamaria, S., \& Hassan Montero, Y. (2013). Análisis y evaluación de sitios web universitarios españoles a partir del proceso de Bolonia. Escola de Ciencia da Información da UFMG. https://doi.org/10.1590/S141399362013000400006

OSCE, \& MEF. (2016). Catalogo Único de Bienes, Servicios y Obras. Jesús María: OSCE. Recuperado de https://bit.ly/2IfZzeV

Presidencia de Consejo de Ministros. (2013). Política Nacional de Gobierno Electrónico 2013- 2017. Lima: Diario Oficial El Peruano. Recuperado de https://bit.ly/2yzFIYE

Presidencia de Consejo de Ministros. (2017a). Declaran el Año 2017 como el Año del Buen Servicio al Ciudadano. Lima, Perú: Diario Oficial El Peruano. Recuperado de https://bit.ly/2K2QKLC

Presidencia de Consejo de Ministros. (2017b). Lineamientos para la implementación del Portal de Transparencia Estándar en las entidades de la Administración Pública. Lima, Perú: Diario Oficial El Peruano. Recuperado de https://bit.ly/2JXu7I5

Robayo Valencia, M. A. (2017). Gobierno Electrónico en el Ecuador: Análisis de su implementación en el marco del Plan Nacional 2014 - 2017. Quito: Universidad Politécnica Salesiana. Recuperado de https://bit.ly/2K79Xeh

Sandoval-Almazan, R., \& Valle-Cruz, D. (2017). Open Innovation, Living Labs and Public Officials: The Case Mapaton in México. New Dheli AA, India: ACM Digital Library. https://doi.org/10.1145/3047273.3047308

SeGDI. (2016). Aprueban Modelo de Gestión Documental en el marco del Decreto Legislativo No 1310. Lima, Perú: Diario Oficial El Peruano. Recuperado de https://bit.ly/2riOGUl

SeGDI. (2017). Sistema Nacional de Informática. Presidencia de Consejo de Ministros. Recuperado de https://bit.ly/2KavShs

Serrano Santoyo, A., \& Martínez, E. (2003). La brecha digital: Mitos y Realidades. México: Universidad Autónoma de Baja California. Recuperado de http://www.uabc.mx/

SGP. (2015). Manual para Mejorar la Atención a la Ciudadanía en las entidades de la Administración Pública. Lima: Presidencia de Consejo de Ministros. Recuperado de https://bit.ly/2lmms5g 
Villoria, M., \& Ramírez A., Á. (2013). Los modelos de gobierno electrónico y sus fases de desarrollo: Un análisis desde la teoría política. México: Centro de Investigación y Docencia Económicas. Recuperado de https://bit.ly/2teUWNN 


\section{PARA CITAR EL ARTÍCULO INDEXADO.}

Alvarado Aguirre, P., Cevallos Vásquez, P., Moran Villamarin, E., \& Preciado Ortiz, L. (2019). Modelo de gobierno electrónico peruano: servicios para el ciudadano en los portales de $\quad \operatorname{los}$ gobiernos regionales. Visionario Digital, 3(3), 100-116. https://doi.org/10.33262/visionariodigital.v3i3.656

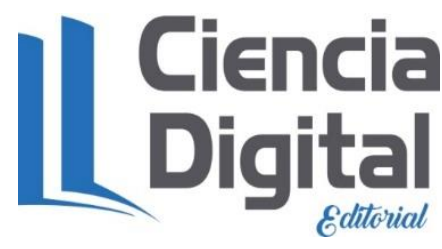

El artículo que se publica es de exclusiva responsabilidad de los autores y no necesariamente reflejan el pensamiento de la Revista Visionario Digital.

El artículo queda en propiedad de la revista y, por tanto, su publicación parcial y/o total en otro medio tiene que ser autorizado por el director de la Revista Visionario Digital.
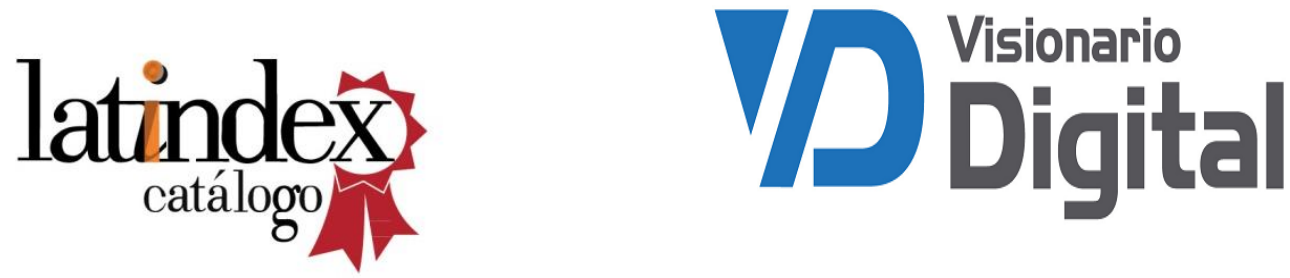